Document downloaded from:

http://hdl.handle.net/10251/82362

This paper must be cited as:

Crites, CL.; Lima, FM.; Marín García, ML.; Netto Ferreira, JC.; Impellizzeri, S.; HallettTapley, GL.; Scaiano, JC. (2016). Tetrahydropyranyl Protection and Deprotection of Alcohols using Niobium Phosphate as a Brønsted Acid Catalyst. Canadian Journal of Chemistry. 94(8):712-714. doi:10.1139/cjc-2015-0538.

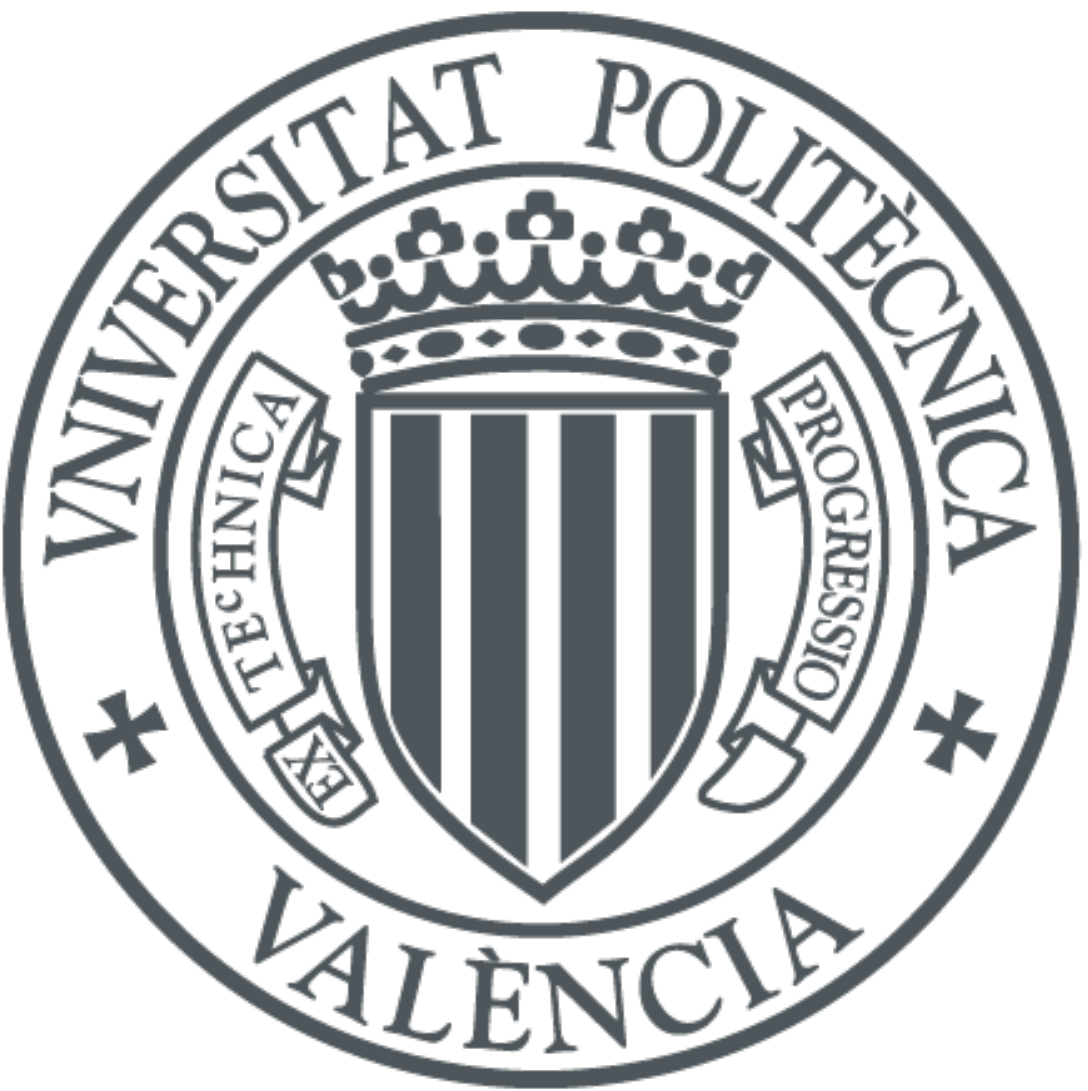

The final publication is available at

http://doi.org/10.1139/cjc-2015-0538

Copyright NRC Research Press (Canadian Science Publishing)

Additional Information 


\title{
Tetrahydropyranyl Protection and Deprotection of Alcohols using Niobium Phosphate as a Brønsted Acid Catalyst
}

Charles-Oneil L. Crites, ${ }^{a}$ Felipe Matos Lima, ${ }^{a}$ M. Luisa Marin,,${ }^{\text {a,b }}$ Jose Carlos Netto-Ferreira, ${ }^{\mathrm{a}, \mathrm{c}}$ Stefania Impellizzeri, ${ }^{a}$ Geniece L. Hallett-Tapley ${ }^{\mathrm{d}}$ and Juan C. Scaiano*a

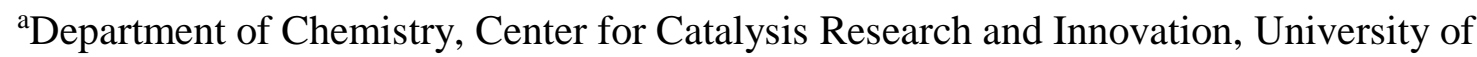
Ottawa, 10 Marie Curie, Ontario K1N 6N5, Canada

${ }^{\mathrm{b}}$ Instituto Universitario de Tecnologia Quimica (UPV-CSIC) Universitat Politècnica de València, Avenida de los Naranjos s/n, 46022 Valencia, Spain

${ }^{\mathrm{c}}$ Instituto Nacional de Metrologia, Qualidade e Tecnologia (INMETRO), Xerem - Duque de Caxias, Rio de Janeiro, CEP: 25520-020, Brazil

${ }^{\mathrm{d} D e p a r t m e n t ~ o f ~ C h e m i s t r y, ~ S t . ~ F r a n c i s ~ X a v i e r ~ U n i v e r s i t y, ~ P . O . ~ B o x ~ 5000, ~ A n t i g o n i s h, ~ N o v a ~}$ Scotia, B2G 2W5, Canada

\begin{abstract}
Niobium phosphate $\left(\mathrm{NbPO}_{4}\right)$ was used as a heterogeneous catalyst in the protection of a series of aliphatic alcohols as tetrahydropyranyl (THP) derivatives. Protection of primary alcohols using this material with strong Brønsted acid sites was achieved in good yields (75-94\%), while moderate values were obtained in the protection of secondary and tertiary alcohols (50-84\%), largely dependent on the steric hindrance in the proximity of the hydroxyl moiety. $\mathrm{NbPO}_{4}$ also catalyzed deprotection of THP ethers derived from primary alcohols and phenol with moderate yields (65-99\%).
\end{abstract}




\section{Introduction}

Protecting groups are important tools in organic synthesis. In particular, dihydropyran (DHP) has been widely employed in the protection of alcohols and phenols to yield the corresponding THP ethers (Table 1), ${ }^{[1]-[2]}$ especially due to the robust and unreactive nature of the resulting ethers under a variety of reaction conditions, notably towards strongly basic media, hydrides, acylating and alkylating agents. ${ }^{[3]}$ Protection of alcohols and phenols with DHP has been widely examined in the past under a number of catalytic conditions, both homogeneous and heterogeneous. Homogenous catalysts, such as $p$-TsOH, ${ }^{[4]} \mathrm{Bi}(\mathrm{OTf})_{3} \cdot \mathrm{H}_{2} \mathrm{O},{ }^{[3]} \mathrm{CuCl}_{2}{ }^{[5]}$ and $p$ toluenesulfonic acid, ${ }^{[6]}$ have been reported. Heterogeneous-derived catalytic pathways have gained considerable attention due the advantageous ease of separation and catalyst reusability, as compared with their homogeneous counterparts. With regards to the THP protection of alcohols, catalysts such as Amberlyst-15, ${ }^{[7]}$ natural Kaolinitic Clays, ${ }^{[8]}$ sepiolite,${ }^{[9]} \mathrm{HY}$ zeolites $^{[10]}$ and $\mathrm{ZnCl}_{2}$ supported on alumina ${ }^{[11]}$ have been successfully used. When considering the deprotection of THP-ehters both homogenous ${ }^{[3,12]}$ and heterogeneous ${ }^{[9,13]}$ approaches have also been used in the past.

Recently, $\mathrm{NbPO}_{4}$ has been employed as an heterogeneous catalyst for the dehydration of fructose, ${ }^{[14]}$ the esterification of fatty acids, ${ }^{[15]}$ the oxidation of benzyl alcohols ${ }^{[16]}$ and the benzylation of anisoles. ${ }^{[17]}$ Due to its considerable Brønsted acidity $\left(\mathrm{H}_{0} \sim-8.6 \text { to }-5.6\right)^{[18]}$ and its heterogeneous nature, $\mathrm{NbPO}_{4}$ warrants investigation for protection-deprotection of alcohols.

Our interest in niobium compounds relates to the fact that this rare metal is produced almost exclusively by Canada and Brazil and used mostly as an additive to steel in order to modify its 
mechanical properties. ${ }^{[19]}$ We believe that applications in the field of catalysis hold the potential for better value-added uses of niobium and some of our recent contributions reflect this interest, as well as collaborative projects between Canada and Brazil.

Given these known properties and the benefits of heterogeneous catalysis, this contribution discusses the use of $\mathrm{NbPO}_{4}$ as a catalyst in this important class of organic reactions, using THP as the primary protective group. To the best of our knowledge, this is the first time $\mathrm{NbPO}_{4}$ has been employed as a catalyst for organic protection-deprotection chemistry.

\section{Experimental}

Materials and instrumentation. Phenol (re-distilled 99\%+), 1-pentanol (99\%+), cinnamyl alcohol (98\%), 3,4-dihydro-2H-pyrane (DHP; 97\%), phenethyl alcohol (99\%), benzyl alcohol

anhydrous $\quad(99.8 \%), \quad$ 1-phenyl-1-propanol $\quad(\geq 97 \%), \quad$ 2-phenyl-2-propanol $\quad(97 \%), \quad 1$ methylcyclohexanol (96\%), tert-butanol (99.5\%+), cyclohexanol (99\%) and dimethyl sulfone $(98 \%)$ were purchased from Sigma-Aldrich and used as received. $\mathrm{NbPO}_{4}$ was generously provided by CBMM (Companhia Brasileira de Metalurgia e Mineração). ACS grade methanol and dichloromethane were purchased from Fisher Scientific and used as received. NMR spectra were recorded using a Bruker Avance $400 \mathrm{MHz}$ in $\mathrm{CDCl}_{3}$.

General procedure for the protection of alcohols with DHP. To a solution of the alcohol (10 mmol) in dichloromethane (25 mL) 3,4-dihydro-2H-pyrane (13 mmol, 1.3 equivalent) was added, followed by $\mathrm{NbPO}_{4}(100 \mathrm{mg})$. The reaction was monitored using GC-MS and, upon completion, the catalyst was separated from the reaction mixture by centrifugation. The solvent and the excess DHP were removed from the reaction mixture by rotary evaporation. The purity 
of the product was examined by ${ }^{1} \mathrm{H}$ and ${ }^{13} \mathrm{C}$ NMR. Compound $\mathbf{1 0}$ was purified by column chromatography $\left[\mathrm{SiO}_{2}\right.$ : Hexanes/EtOAc (4:1)].

General procedure for the deprotection of THP ethers. To a solution of the THP ether ( $3 \mathrm{mmol})$ in refluxing $\mathrm{MeOH}(25 \mathrm{~mL}) \mathrm{NbPO}_{4}(25 \mathrm{mg})$ and dimethyl sulfone $(3.5 \mathrm{mmol})$ were added. The reaction was monitored by GC-MS and then the catalyst was separated from the reaction mixture by centrifugation. The solvent was eliminated under reduced pressure and the crude analyzed by ${ }^{1} \mathrm{H}$ and ${ }^{13} \mathrm{C}$ NMR using dimethyl sulfone as an internal standard.

Recyclability Experiments. The recyclability of the $\mathrm{NbPO}_{4}$ catalyst was examined in both the DHP protection of benzyl alcohol and the corresponding THP deprotection. The initial protection and deprotection trials were carried out using the experimental procedures described above. Following the initial trial, the NbPO4 catalyst was recovered and washed with $\mathrm{CH}_{2} \mathrm{Cl}_{2}$ for the DHP protection and $\mathrm{CH}_{3} \mathrm{OH}$ for the THP deprotection. This procedure was repeated a total of four times to remove any adsorbed materials from the catalyst surface of the catalyst. After the final wash, the supernatant was decanted and the solid left to dry overnight. The recovered $\mathrm{NbPO}_{4}$ was weighted and the reagents scaled down to ensure a consistent substrate/catalyst ratio in subsequent reuse trials.

\section{Results and Discussion}

The experimental procedure of the protection of alcohols is relatively straightforward. $\mathrm{NbPO}_{4}$ was added to a solution of the alcohol substrate and THP in dichloromethane, and the reaction mixture was stirred at room temperature and monitored by thin layer chromatography or GCMS. The catalyst was then removed simply by centrifugation at $3000 \mathrm{rpm}$ and the excess of DHP was eliminated under reduced pressure. 
Table 1. $\mathrm{NbPO}_{4}$-catalyzed formation of THP ethers from a series of alcohol and phenol substrates.

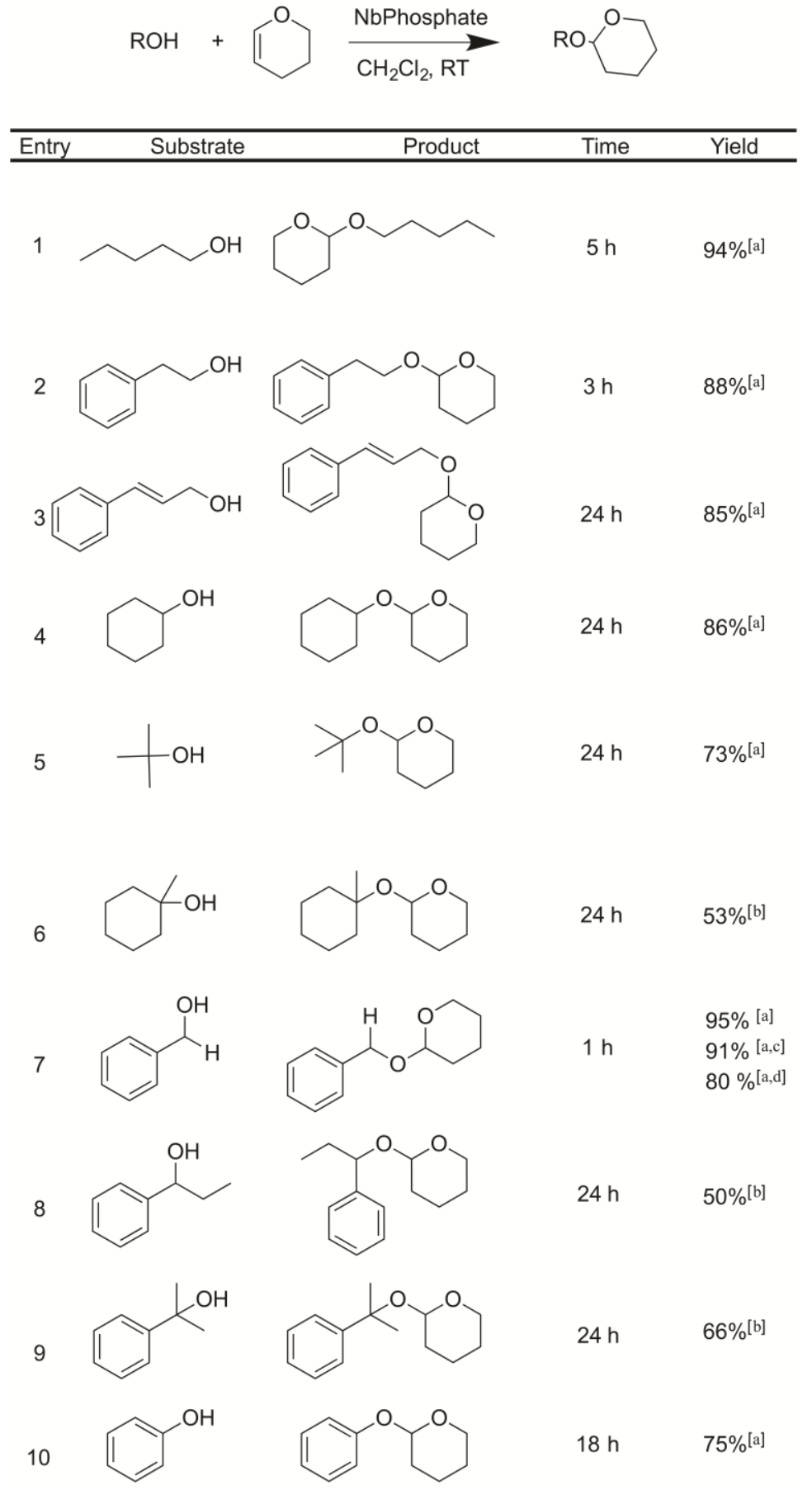

assolated yield. ${ }^{\text {b }}$ Determined by ${ }^{1} \mathrm{H}$ NMR spectroscopy. ${ }^{\mathrm{c}}$ Reuse trial $1 .{ }^{\mathrm{d}}$ Reuse trail 2. 
The isolated yields obtained for the formation of THP ethers from a series of organic alcohols are presented in Table 1. The yields obtained show a clear dependence on the steric hindrance suffered by the reactive $-\mathrm{OH}$ center. Specifically, protection of primary alcohols $\mathbf{1 - 3}$ and 7 afforded the highest isolated yields of the corresponding THP-ether, ranging from $85 \%$ to $94 \%$. Accordingly, the yield of the protected product was found to decrease concomitantly with the substitution of the alcohol functionality. This trend is clearly observed when comparing the values obtained for a structurally comparable secondary and tertiary alcohol. Thus, the protection of cyclohexanol (4) and 1-methyl-cyclohexan-1-ol (6) illustrates a considerable decrease in the efficacy of the $\mathrm{NbPO}_{4}$-catalysed protection pathway (from $86 \%$ to $53 \%$, respectively) with increasing substitution of the alcohol moiety. The same general trend was observed when comparing the yields for entries $\mathbf{7}$ and $\mathbf{8}$ where increased substitution of the alcohol translates into a $38 \%$ reduction in the yield of product obtained. The protection of these sterically hindered alcohols also required longer reaction times. Indeed, increased steric hindrance in close proximity to the $\mathrm{OH}$ functionality will partially inhibit its addition to the carbocation that is initially formed in the reaction upon the protonation of DHP in a similar fashion to the acid catalyzed hydration of alkenes. Interestingly, $\mathrm{NbPO}_{4}$ has also been successfully employed in the THP protection of phenol (6) in $75 \%$ yield over moderate reaction times (18 hours) (see Table 1). The recyclability of the catalyst was determined by reusing the catalyst in two separate trials in the protection of benzyl alcohol. The initial isolated yield (95\%) was found to decrease marginally to $91 \%$ after one reuse and to $80 \%$ after a second recyclability trial. After three reuses, the $\mathrm{NbPO}_{4}$ catalyst still demonstrated considerable acidic activity, indicating that this heterogeneous material may be an economical and recyclable means of promoting DHP alcohol protections. 
Methods used for the deprotection of THP ethers generally employ strongly acidified reaction media and long reaction times. ${ }^{[3]}$ Therefore, a mild but efficient method to selectively deprotect THP ethers in the presence of other sensitive functional groups has yet to be achieved. To this end, $\mathrm{NbPO}_{4}$ was investigated as a potential alternative to catalytically deprotect THP ethers and restore the corresponding alcohol by acidic hydrolysis based on its strong Brønsted acid nature using relatively mild reaction conditions. In fact, deprotection of THP ethers was achieved in good yields under stirring in refluxing methanol.

Table 2. $\mathrm{NbPO}_{4}$-catalyzed deprotection of THP ethers substrates.
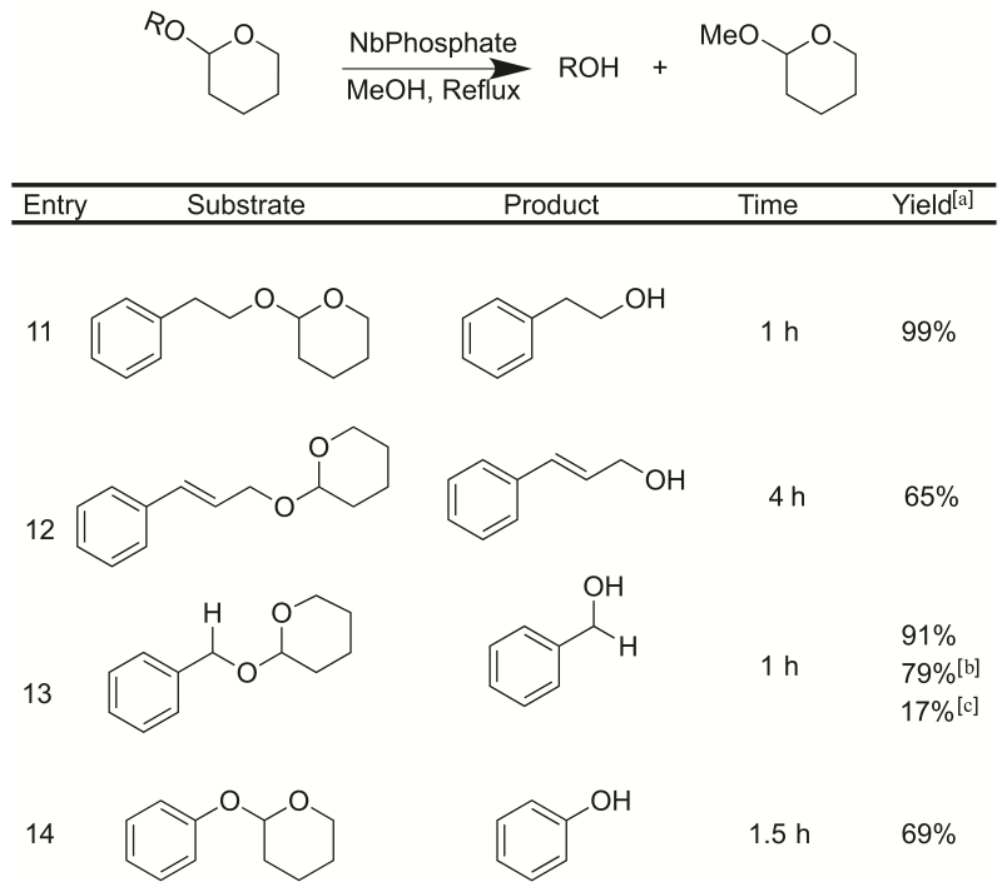

${ }^{a}$ Yields obtained by NMR using dimethyl sulfone as internal standard ${ }^{\text {b}}$ Reuse trial $1 .{ }^{\mathrm{c}}$ Reuse trial 2. 
The results obtained for the deprotection of THP ethers 11-14 are presented in Table 2. The yield for deprotection of THP ethers formed from primary alcohols (entries 11-12) resulted in large alcohol yields (65\% to 99\%). Compound $\mathbf{1 4}$ was also successfully deprotected to phenol using $\mathrm{NbPO}_{4}$ with a yield of $69 \%$ in 1.5 hours. Importantly, $\mathrm{NbPO}_{4}$ catalyzed protection is better, both in temperature and time, when compared to previous deprotection studies using $\mathrm{Bi}(\mathrm{OTf})_{3} \bullet \mathrm{H}_{2} \mathrm{O}$ as a homogeneous catalyst. ${ }^{[3]}$ As was examined with the DHP protection experiments, the reusability of $\mathrm{NbPO}_{4}$ in THP deprotection was also examined. The initial yield in the $\mathrm{NbPO}_{4}$-initiated deprotection of THP benzyl alcohol was $91 \%$. This yield decreased to $79 \%$ after only one reuse and further diminished to a mere $17 \%$ after a second trial. These results suggest that $\mathrm{NbPO}_{4}$ is not as efficiently recycled in THP deprotection process.

$\mathrm{NbPO}_{4}$ holds a clear advantage to other catalysts that have been employed in both DHP protection and THP deprotection studies. Recyclability experiments using $\mathrm{NbPO}_{4}$ clearly demonstrate that the heterogeneous nature of the niobium-derived catalyst allows for the recovery and reuse of the material in several subsequent trials, lending to increased economic advantage of this material as compared to the traditional homogeneous catalysts. $\left.{ }^{[2,} 4-6\right]$ Comparison with other heterogeneous catalysts is difficult, as yields are comparable between $\mathrm{NbPO}_{4}$ and these alternatives. However, literature precedents using other heterogeneous supports have lacked study on the recyclability of these materials. ${ }^{[8-11]}$ Given $\mathrm{NbPO}_{4}$ is a very much a Canadian resource, the development and application of this material within common, catalytically induced organic transformations is important for both economic and environmental sustainability. 


\section{Conclusion}

The protection and deprotection of alcohols using tetrahydropyranyl (THP) derivatives was performed using $\mathrm{NbPO}_{4}$ as catalyst. It was shown that the protection of alcohol was obtained in high yield especially in the case of primary alcohols reflecting the reduced steric hindrance as compared to secondary and tertiary alcohols. It was also shown that phenol could be protected using the same protocol. The deprotection of primary alcohols and phenol also showed high yields. Finally, exceptional recyclability of the $\mathrm{NbPO}_{4}$ catalyst in benzyl alchol DHP protection trials, followed by moderate reusability in THP deprotection demonstrates the robust and sustainable acid catalytic nature of this abundant Canadian resource. 


\section{$\underline{\text { References }}$}

[1] W. E. Parham, E. L. Anderson, J. Am. Chem. Soc. 1948, 70, 4187-4189.

[2] T. W. Green, P. G. M. Wuts, Protection Groups in Organic Synthesis 3rd Edition, John Willey \& Sons, Inc, New York, 1999.

[3] J. R. Stephens, P. L. Butler, C. H. Clow, C. Oswald, R. C. Smith, R. S. Moham, Eur. J. Org. Chem. 2003, 3827-3831.

[4] J. H. van Boom, C. B. Herschied, Synthesis 1973, 169-170.

[5] U. T. U.T. Bhalerao, K. Job Davis, B. Vial Rao, Synth. Commun. 1996, 26, 3081-3085.

[6] A. Miysahita, A. Yoshikoshi, P. A. Grieco, J. Org. Chem. 1977, 42, 3772-3774.

[7] A. Bongini, G. Cardillio, M. Orena, S. Sandri, Synthesis 1979, 9, 618-620.

[8] T. T. Upadhyda, T. Daniel, A. Sudalai, T. Ravindranathan, K. R. Subu, Synth. Commun. 1996, 26, 4539-4555.

[9] J. M. Campelo, A. Garcia, F. Lafont, D. Luna, J. M. Marinas, Synth. Commun. 1994, 24, 1345-1350.

[10] P. Kumar, C. U. Dinesh, R. S. Reddy, B. Pandey, Synthesis 1993, 11, 1069-1070.

[11] B. C. Ranu, M. Saha, J. Org. Chem. 1994, 59, 8269-8270.

[12] aJ. Liu, C. H. Wong, Tetrahedron Lett. 2002, 43, 4037-4039; bJ. Wang, C. Zhang, Z. $\mathrm{Qu}$, Y. Hou, B. Chen, P. Wu, J. Chem. Research 1999, 294-295.

[13] aP. Lazslo, S. Hoyer, Synthesis 1986, 24, 655-656; bG. Sartori, R. Ballini, F. Bigi, G. Bosica, R. Maggi, P. Righ, Chem. Rev. 2004, 104, 199-250.

[14] P. Carniti, A. Gervasini, S. Biella, A. Auroux, Catal. Today 2006, 118, 373-378.

[15] I. A. L. Bassana, D. R. Nascimentoa, R. A. S. San Gilb, M. Pais da Silvac, I., C. R. Moreirac, W. A. Gonzalezd, A. C. Faro Jr, Onfroye, T, E. R. Lachtera, Fuel Process Technol 2013, 106, 619-624.

[16] M. C. Reisa, S. D. T. Barrosa, E. R. Lachtera, R. San Gilb, A.S., J. H. Floresc, M. I. Pais da Silvac, T. Onfroy, Catal. Today 2012, 192, 117-122.

[17] A. S. Rocha, A. M. S. Forrester, M. H. C. de la Cruz, C. T. da Silva, E. R. Lachter, Catal. Commun. 2008, 9, 1959-1965.

[18] S. Impellizzeri, S. Simoncelli, C. Fasciani, M. L. M. Luisa Marin, G. L. Hallett-Tapley, G. K. Hodgson, J. C. Scaiano, Catal. Sci. Technol. 2015, 5, 169-175.

[19] A. W. Den Boer, D. V. Malakhov, Can. Metall. Q. 2014, 53, 423. 